\title{
不同基因型羊草数量性状的可塑性及遗传分化
}

\author{
杨 雪 申俊芳 赵念席 ${ }^{*}$ 高玉葆
}

南开大学生命科学学院, 天津 300071

摘 要 为了深入探讨植物对环境变化的适应机制, 该文以内蒙古草原区羊草(Leymus chinensis)不同基因型为对象, 在人工 控制条件下, 研究了羊草基因型、刈割、干旱及其交互作用对羊草11个数量性状的影响。结果显示: (1)所观测的11个性状(光 系统II光化学效率、最大净光合速率、蒸腾速率、比叶面积、相对生长速率、分真增长数、地上及地下生物量、叶总酚浓度、 根非结构性碳水化合物总量和根冠比)受环境因素(干旱、刈割或两者交互)影响显著, 表明该物种具有较强的表型可塑性; 且 在4种环境条件(对照、刚割、干旱、刚割干旱)下, 不同基因型羊草的反应规范并不一致, 其中, 最大净光合速率、蒸腾速率、 比叶面积、相对生长速率、叶总酚浓度和根非结构性碳水化合物总量受环境和基因型交互作用影响显著, 表明这些性状的表 型可塑性具有一定的遗传基础。(2)对同一环境条件下, 不同基因型间的性状进行分析显示, 分蒕增长数、地下生物量和根非 结构性碳水化合物含量在4种环境条件下均未检测到基因型间的差异; 而其余8个性状在基因型间的差异显著，表明这些性状 的差异具有一定的遗传基础, 其中, 与生长相关的 6 个性状的遗传力 $\left(H^{2}\right)$ 较高, 均大于 0.5 , 而叶总酚浓度和根冠比仅在刈割干 旱条件下检测出显著差异, $H^{2}$ 分别为 0.145 和 0.202 。这些实验结果为理解羊草这一重要物种在内蒙古草原区的广泛分布提供 了适应机制方面的数据支持, 为合理预测未来气候变化对该物种的影响提供了科学依据, 为合理利用和保护该物种及其生态 系统提供了理论依据。

关键词 羊草; 基因型; 适应; 遗传分化; 表型可塑性; 反应规范

引用格式: 杨雪, 申俊芳, 赵念席, 高玉葆 (2017). 不同基因型羊草数量性状的可塑性及遗传分化. 植物生态学报, 41, 359-368. doi: 10.17521/cjpe. 2015.0257

\section{Phenotypic plasticity and genetic differentiation of quantitative traits in genotypes of Leymus chinensis}

YANG Xue, SHEN Jun-Fang, ZHAO Nian-Xi*, and GAO Yu-Bao

College of Life Sciences, NanKai University, Tianjin 300071, China

\section{Abstract}

Aims Adaptation mechanisms of plants to environment can be classified as genetic differentiation and phenotypic plasticity (environmental modification). The strategy and mechanism of plant adaptation is a hot topic in the field of evolutionary ecology. Leymus chinensis is one of constructive species in the Nei Mongol grassland. Particularly, Leymus chinensis is a rhizomatous and clonally reproductive grass, a genotype that can play an important role in the community. In this study, we aimed to (1) investigate the phenotypic plasticity of L. chinensis under different conditions, and (2) test the genetic differentiation and reaction norms (the relationship between the environment and the phenotype of an individual or a group of individuals) under four environmental conditions among different genotypes of L. chinensis.

Methods Ten genotypes of L. chinensis were randomly selected. Under the control condition, we studied the effects of genotype, defoliation, drought and their interactions on 11 quantitative traits of growth (8 traits including photochemical efficiency of photosystem II, maximum net photosynthetic rate, transpiration rate, specific leaf area, relative growth rate, the number of tillers increased, aboveground and underground biomass growth), defense (total phenol concentration of leaf) and tolerance (non-structural carbohydrate content of root, root/shoot ratio) of $L$. chinensis. We studied the phenotypic plasticity, genetic differentiation and reaction norms mainly through tested the effect of environment and genotype on these traits.

Important findings First, all 11 traits showed obvious phenotypic plasticity (i.e., significant effect of drought, defoliation and their interactions). The expression of 10 genotypes of $L$. chinensis was divergent under different

收稿日期Received: 2015-07-07 接受日期Accepted: 2016-09-21

* 通信作者Author for correspondence (E-mail: zhaonianxi@nankai.edu.cn ) 
environmental conditions. Interactions of genotype and environment significantly affected the maximum net photosynthetic rate, transpiration rate, specific leaf area, relative growth rate, total phenolic concentration of leaf, and total non-structural carbohydrate content of root. This indicated that the phenotypic plasticity of these five traits exhibited genetic differentiation. Second, the increase of number of tillers, belowground biomass and non-structural carbohydrate content of root did not show genetic differentiation under the same condition. The other eight traits showed significantly genetic differentiation, and the heritabilities $\left(H^{2}\right)$ of six traits related to growth were higher than 0.5 . The leaf total phenol concentration and root/shoot ratio showed genetically differentiation only under the drought and defoliation condition, with the heritabilities being 0.145 and 0.201 , respectively. These results explained why L. chinensis widely distributed in the Nei Mongol grassland, and provided genetic and environmental basis for related application and species conservation in this grassland ecosystem.

Key words Leymus chinensis; genotype; adaptation; genetic differentiation; phenotypic plasticity; reaction norm

Citation: Yang X, Shen JF, Zhao NX, Gao YB (2017). Phenotypic plasticity and genetic differentiation of quantitative traits in genotypes of Leymus chinensis. Chinese Journal of Plant Ecology, 41, 359-368. doi: 10.17521/cjpe.2015.0257

生物在生长发育过程中不可避免地经历外部环 境的变化，生物对环境的适应机制主要有两种：遗 传分化 (genetic differentiation) 和表型可塑性 (phenotypic plasticity) (Liefting et al., 2009)。前者指 通过遗传的改变来产生可稳定遗传的表型变异 (Hedrick, 2005); 后者则通过环境饰变来产生依赖 于环境的表型变异(Sultan, 2000)。同一基因型在不 同环境下产生的一系列表型被称为反应规范 (reaction norm) (Miner et al., 2005), 反应规范在同 一物种内不同基因型间也会存在显著差异(环境因 素×基因型作用显著), 即反应规范的遗传分化或表 型可塑性的遗传分化(Mallitt et al., 2010)。表型可塑 性与遗传分化是生物适应环境变化的两种不同但并 不互相排斥的策略, 同一物种的适应可以既有表型 可塑性又有遗传分化, 两者对环境变化适应的相对 重要性与具体性状、生态因子等有关(Sultan, 1995)。 对物种适应机制的深入研究不仅能够了解该物种对 环境的适应策略和维持机制，而且能够对未来全球 变化下物种的进化潜力进行正确预测, 因此, 该 方面的研究一直以来都是种群生态学及种群遗传学 研究的热点问题(Lande, 2009; Draghi \& Whitlock, 2012)。

随着种群遗传学研究的不断深入, 人们发现种 内基因型间的性状变异不仅对种群的适应性进化具 有重要作用, 而且能发挥物种水平的作用, 对群落 乃至生态系统进程也具有重要影响(Crutsinger et al., 2009; Cook-Patton et al., 2011)。如不同基因型的大 叶藻(Zostera marina)不仅在生物量方面存在差异, 而且在铵态氮和硝态氮的利用方面也存在差异, 不 同基因型表现出养分利用的分化(Hughes et al.,
2009); 不同基因型的美洲山杨(Populus tremuloides) 对食草动物的防御和耐受存在差异, 且这种差异是 由遗传因素决定的(Stevens et al., 2007)。因此, 关于 重要物种基因型间差异的研究不仅能反映植物适应 策略, 而且对该物种在群落中的重要作用的正确评 估也有一定的积极意义(Johnson \& Agrawal, 2005; Kanaga et al., 2008; Hughes et al., 2009; Le Corre \& Kremer, 2012; Strasburg et al., 2012)。

近半个世纪, 特别是 20 世纪 80 年代以来, 全球 气候变化和人类活动加剧引起生态系统退化加重。 草原作为重要的生态系统, 退化尤为严重, 草原群 落的恢复和重建成为亟须解决的问题(Joshi et al., 2006)。而这一问题的解决主要集中在草原建群种和 优势种对环境变化的适应机制和适应策略的研究上, 因为优势物种对生态系统有重要的指示和调节作用 (Seliskar et al., 2002)。羊草(Leymus chinensis)作为内 蒙古草原的建群种, 具有生态幅广、适应性强等特 点, 如早期的研究发现, 羊草种群间遗传分化显著 (胡宝忠等, 2001; 刘惠芬等, 2004a, 2004b), 数量性 状(生长和生理性状)存在显著的差异(Wang \& Gao, 2001; 李红等, 2004; 李海燕等, 2011); 但针对这一 重要物种适应机制的探讨并没有深入展开。另外, 羊草作为根茎型禾草, 通过克隆繁殖, 某些特定的 基因型在群落中也能起到很重要的作用。最近有研 究发现，羊草不同基因型间具有类似于物种间的生 态位互补效应(申俊芳等, 2015), 但这种互补效应是 否有遗传学基础并没有得到证实，且不同基因型间 性状的差异是否能够反映适应策略的不同也未见报 道。因此, 基于内蒙古草原区一直经历严重的放牧 压力和水分分布不均匀的特点, 本研究选择不同基 www.plant-ecology.com 
因型羊草为研究对象, 并进行刈割、干旱处理，探讨： (1)随环境条件变化, 同一基因型羊草的数量性状的 表型可塑性及对应的适应策略; (2)性状的反应规范 在基因型间是否存在显著差异; (3)同一环境条件下, 羊草数量性状是否受基因型影响显著, 并重点关注 胁迫环境下与防御和耐受相关的性状。所得结果不 仅能够了解羊草对环境变化的适应机制及策略, 为 合理预测未来气候变化对该物种的影响提供理论基 础, 而且对羊草种群及其所在群落的合理利用、保 护和资源开发也具有重要的意义。

\section{1 材料和方法}

\section{1 实验材料}

本实验所用羊草为 2010 年于内蒙古锡林浩特 市阿巴嘎旗典型草原西界 $\left(43.90^{\circ} \mathrm{N}, 115.34^{\circ} \mathrm{E}\right)$ 采集 的羊草基株, 利用 ISSR 分子标记 $(\mathrm{AG})_{7} \mathrm{~T}$ 和 $(\mathrm{CA})_{6} \mathrm{~A}$ 确定基因型(王丹, 2004), 并进行编号。在同样的条 件下培养以去除母体效应，通过根茎无性繁殖得到 同一基因型的大量分藓。从已有的基因型中随机选 择10个基因型( $\mathrm{Y} 7 、 \mathrm{Y} 8 、 \mathrm{Y} 18 、 \mathrm{Y} 23 、 \mathrm{Y} 24 、 \mathrm{Y} 25$ 、 $3 / 0 、 3 / 1 、 3 / 25 、 4 / 6)$ 进行实验。

\section{2 实验设计}

本实验采用三因素实验设计，即基因型 $(10) \times$ 刈割(无刈割(De-)、刚割( $\mathrm{De}+$ ) $)$ ×水分(非干旱(Dr-)、 干旱 $(\mathrm{Dr}+))$, 每个处理 6 个重复。刚割为轻度刈割, 留茬 $15 \mathrm{~cm}$; 非干旱处理 $(\mathrm{Dr}-)$ 的土壤含水量保持在 $15 \%-25 \%$; 干旱(Dr+)处理保持在 $4 \%-9 \%$ 之间。即本 实验共有 4 种环境条件：对照 (De-Dr-)、刚割 $(\mathrm{De}+\mathrm{Dr}-)$ 、干旱 $(\mathrm{De}-\mathrm{Dr}+)$ 、刚割干旱 $(\mathrm{De}+\mathrm{Dr}+)$ 。

2013年1月14日, 选择生长状态良好、大小一 致的羊草分菜，按基因型进行标记后统一去除根 茎并修剪使地上部高度为 $15 \mathrm{~cm}$, 地下根系长度为 $15 \mathrm{~cm}$, 地上部叶片数为 4 片。将这些分繤移栽到装 满湿润蛭石的直径 $20 \mathrm{~cm}$ 的塑料花盆中, 每盆2株。 对移栽1周内死去的分蘖，重新种植。经过一段时 间的恢复生长后，在2013年4月18日，对实验种群 进行干旱处理直至2013年10月14日实验结束, 并 于2013年4月18日、7月4日、9月4日进行3次刈割。 实验期间，无营养添加，随时除杂草，无高温和遮 阳等胁迫; 所有盆随机摆放, 每 2 周移动位置 1 次以 避免位置效应。实验在南开大学生命科学学院网室 中进行。

\section{3 指标测定}

本研究选择了 11 个指标进行测定, 包括 8 个与 植物生长相关的指标, 光系统II (PSII) 光化学效率 $\left(F_{\mathrm{v}} / F_{\mathrm{m}}\right)$ 、最大净光合速率 $\left(P_{\mathrm{nmax}}\right)$ 、蒸腾速率 $\left(T_{\mathrm{r}}\right)$ 、比 叶面积 $(S L A)$ 、相对生长速率 $(R G R)$ 、分蓝增长数、 地上部及地下部生物量; 1 个与植物防御相关的指 标，叶总酚浓度; 以及 2 个与植物耐受相关的指标, 根非结构性碳水化合物(TNC)总量和根冠比。具体 测定方法如下:

$F_{\mathrm{v}} / F_{\mathrm{m}}$ : 于 2013 年 4 月 20 日上午, 选择每个羊草 基株上从内向外第二片完全展开的叶片, 暗处理 20 $\min$ 后使用便携式叶绿素苂光仪 (Handy PEA, Hansatech Instruments, King's Lynn, UK)进行测定。

$P_{\mathrm{nmax}}$ 和 $T_{\mathrm{r}}$ : 选择晴朗无云的上午(2013年4月 21 日至23日), 使用便携式光合仪(LI-6400, LI-COR, Lincoln, USA)选择每个羊草基株的从内向外第二片 完全展开叶，根据研究区晴天日照光强，设置测定 光强为 $800 \mu \mathrm{mol} \cdot \mathrm{m}^{-2} \cdot \mathrm{s}^{-1}$, 叶室温度为 $25{ }^{\circ} \mathrm{C}$, 测定叶 片 $P_{\mathrm{n} m a x}$ 和 $T_{\mathrm{r}}$ 。植物经过刚割后光合能力会有短时的 下降, 这种瞬变反应一般最多维持 $24 \mathrm{~h}$, 此后光合 能力提升, 这个反应在受损和未受损叶片中是相似 的(Richards, 1993)。因此我们选择在刈割 $24 \mathrm{~h}$ 之后测 定 $F_{\mathrm{v}} / F_{\mathrm{m}} 、 P_{\mathrm{nmax}}$ 和 $T_{\mathrm{r}}$ 。

$S L A: 2013$ 年10月10日, 以基株为单位选择从内 向外第二片完全展开的向阳叶片, 利用叶面积仪 (LI-3100，LI-COR，Lincoln，USA)测定叶片面积， $80{ }^{\circ} \mathrm{C}$ 烘干至恒质量后用 $1 / 10000$ 天平称量叶片干质 量。SLA =叶片面积/叶片干质量。

$R G R: R G R=\left(\ln H_{2}-\ln H_{1}\right) /\left(t_{2}-t_{1}\right)$ 。其中, $H_{1}$ 、 $\mathrm{H}_{2}$ 分别为某次刈割后的株高和下一次刈割前(或收 获前)的株高; $t_{1} 、 t_{2}$ 分别为 $H_{1} 、 H_{2}$ 对应的时间; 实验 期间共刏割 3 次，统计分析所用 $R G R$ 为 3 次结果的平 均值。

分藥增长数、地上部、地下部生物量及根冠比: 2013年10月14日, 统计分藥数, 减去初始分等数得 到分茧增长数; 以基株为单位按地上部与地下部收 获植物, $105{ }^{\circ} \mathrm{C}$ 杀青 $30 \mathrm{~min}$ 后, $80{ }^{\circ} \mathrm{C}$ 烘干至恒质量, $1 / 10000$ 天平称质量分别得到地上生物量和地下生 物量干质量, 并计算根冠比。

叶总酚浓度测定采用苯酚比色法(Malinowski et al., 1998); TNC总量测定采用3, 5-二硝基水杨酸 比色法(Da Silveira et al., 1978), 并根据相应的地下 
生物量计算TNC总量。

\section{4 数据处理}

将所得的数据输入Excel进行整理、做图, 使用 SPSS 20.0 (IBM, USA)进行数据统计分析。

利用SPSS 20.0对数据进行正态性检验，对不符 合正态分布的数据进行对数转换使之符合方差分析 的要求。然后利用SPSS 20.0一般线性模型(GLM)中 的多因素方差分析评估多变量基因型 $(\mathrm{G}) 、$ 刈割处理 $(\mathrm{De})$ 、水分处理 $(\mathrm{Dr})$ 以及它们之间的交互作用对所 观测指标平均值影响的显著性。做出不同环境条件 下各个基因型所观测指标的表现，直观地比较不同 基因型的可塑性、反应规范的异同。

本实验使用的是无性系分株，同一基因型重复 间的变异可以代表环境变异, 而基因型间的变异代 表遗传变异。为了估计某一环境条件下遗传及环境 对某一性状影响的大小, 本研究还分别对 4 种环境 条件下基因型间的差异进行了如下分析：(1)使用单 因素方差分析检测基因型对所观测变量的平均值是 否影响显著, 并对基因型影响显著的变量进行广义 遗传力 $\left(H^{2}=\right.$ 遗传方差/总方差(Kanaga et al., 2008)) 的计算来评估基因型间的遗传变异的影响(Toker, 2004)。通常认为, 当 $H^{2}<0.2$ 时, 属于低、弱度遗传 力; $0.2 \leqslant H^{2} \leqslant 0.5$, 为中等遗传力; $H^{2}>0.5$, 为高 强度遗传力(顾万春, 2004)。(2)为了评估基因型间的 变异以及所观测性状对变异的贡献, 使用典则判别 分析(CDA)分别对 4 种环境条件下的 10 个基因型进
行分组并计算各个观测变量的贡献率。已有研究证 明CDA在分析种内变异时是有效的(Hughes et al., 2009)。

\section{2 结果}

\section{1 表型可塑性和反应规范}

基因型、干旱、刚割及其交互作用对不同指标 的影响并不完全相同(表1), 图1比较直观地给出了 10 种基因型羊草在 4 种处理下各个指标的表型可塑 性和反应规范，结果发现：没有哪个基因型在所有 观测的指标中都表现出最佳值; 刈割、干旱及其交 互作用对多数观测指标影响显著(表1) $(p<0.05)$, 即 所有性状均具有表型可塑性, 如则割处理提高 $R G R$ 以及叶总酚浓度(图1E、1I), 干旱处理提高植物根冠 比(图1K)。而基因型与环境因子之间交互作用只对 某些指标影响显著, 如基因型 $\times$ 刈割对 $P_{n \max } 、 T_{\mathrm{r}}$ 、 $R G R$ 、叶总酚浓度和根 $\mathrm{TNC}$ 总量, 基因型 $\times$ 干旱对 $T$ r $S L A 、 R G R$ 、叶总酚浓度和根 $\mathrm{TNC}$ 总量, 以及基 因型 $\times$ 刈割 $\times$ 干早仅对 $R G R$ 具有显著影响 $(p<0.05)$, 表明这些性状的表型可塑性具有遗传学基础。具体 来分析, 相对于对照(De-Dr-), 刈割干旱处理 $(\mathrm{De}+\mathrm{Dr}+)$ 下基因型 $3 / 25$ 中叶总酚浓度(防御相关)显 著升高，而Y24中叶总酚浓度降低(图1I); 相对于对 照(De-Dr-), 刈割处理(De+Dr-)使基因型3/25中根 TNC总量(耐受相关)显著升高，而Y8、Y18无明显变 化(图1J)。

表1 不同基因型羊草表型变异的多因素方差分析及交互作用

Table 1 Multivariate analysis of variance for genotype and phenotypic variations and interactions of genotype and treatments in Leymus chinensis

\begin{tabular}{|c|c|c|c|c|c|c|c|c|c|c|c|c|c|c|}
\hline \multirow[t]{2}{*}{ 性状 Traits } & \multicolumn{2}{|c|}{$\begin{array}{c}\text { 基因型 } \\
\text { Genotype (G) }\end{array}$} & \multicolumn{2}{|c|}{$\begin{array}{c}\text { 刏割 } \\
\text { Defoliation (De) }\end{array}$} & \multicolumn{2}{|c|}{$\begin{array}{c}\text { 干旱 } \\
\text { Drought (Dr) }\end{array}$} & \multicolumn{2}{|c|}{$\mathrm{De} \times \mathrm{Dr}$} & \multicolumn{2}{|c|}{$\begin{array}{l}\text { 基因型 } \times \text { 刈割 } \\
\text { G } \times \text { De }\end{array}$} & \multicolumn{2}{|c|}{$\begin{array}{l}\text { 基因型×干早 } \\
\text { G×Dr }\end{array}$} & \multicolumn{2}{|c|}{$\begin{array}{l}\text { 基因型 } \times \\
\text { 割 } \times \text { 干早 } \\
G \times \operatorname{De} \times \text { Dr }\end{array}$} \\
\hline & $F$ & $p$ & $F$ & $p$ & $F$ & $p$ & $F$ & $p$ & $F$ & $p$ & $F$ & $p$ & F & $p$ \\
\hline $\begin{array}{l}\text { 光系统II光化学效率 Photochemi- } \\
\text { cal efficiency of photosystem II }\end{array}$ & 19.47 & $<0.001$ & 2.649 & 0.105 & 17.19 & $<0.001$ & 0.476 & 0.491 & 1.064 & 0.391 & 0.912 & 0.516 & 1.824 & 0.066 \\
\hline $\begin{array}{l}\text { 最大净光合速率 Maximum net } \\
\text { photosynthetic rate }\end{array}$ & 41.08 & $<0.001$ & 315.7 & $<0.001$ & 7.305 & 0.007 & 0.023 & 0.879 & 9.143 & $<0.001$ & 1.385 & 0.197 & 1.353 & 0.212 \\
\hline 蒸腾速率 Transpiration rate & 4.878 & $<0.001$ & 4.979 & 0.027 & 2.226 & 0.137 & 1.764 & 0.186 & 3.817 & $<0.001$ & 2.805 & 0.004 & 0.881 & 0.543 \\
\hline 比叶面积 Specific leaf area & 3.781 & $<0.001$ & 34.3 & $<0.001$ & 2.916 & 0.089 & 11.62 & 0.001 & 1.231 & 0.278 & 2.207 & 0.023 & 1.843 & 0.063 \\
\hline 相对生长速率 Relative growth rate & 2.829 & 0.006 & 1765 & $<0.001$ & 212.8 & $<0.001$ & 115.1 & $<0.001$ & 6.55 & $<0.001$ & 3.071 & 0.003 & 4.847 & $<0.001$ \\
\hline $\begin{array}{l}\text { 分䡞增长数 The number of tillers } \\
\text { increased }\end{array}$ & 1.842 & 0.073 & 4.488 & 0.037 & 30.15 & $<0.001$ & 24.72 & $<0.001$ & 1.061 & 0.401 & 1.5 & 0.162 & 0.848 & 0.574 \\
\hline 地上生物量 Aboveground biomass & 1.454 & 0.167 & 0.007 & 0.933 & 69.06 & $<0.001$ & 28.66 & 0.001 & 1.116 & 0.353 & 1.848 & 0.062 & 0.635 & 0.767 \\
\hline 地下生物量 Belowground biomass & 2.249 & 0.021 & 1.221 & 0.27 & 21.93 & $<0.001$ & 11.07 & 0.001 & 0.527 & 0.854 & 1.644 & 0.105 & 0.664 & 0.741 \\
\hline $\begin{array}{l}\text { 叶总酚浓度 Total phenolic concen- } \\
\text { tration of leaf }\end{array}$ & 1.624 & 0.111 & 30.62 & $<0.001$ & 0.002 & 0.962 & 48.21 & $<0.001$ & 1.978 & 0.044 & 2.321 & 0.017 & 0.957 & 0.477 \\
\hline $\begin{array}{l}\text { 根非结构性碳水化合物总量 Total } \\
\text { non-structural carbohydrates content } \\
\text { of root }\end{array}$ & 1.480 & 0.157 & 11.09 & 0.001 & 47.13 & $<0.001$ & 16.21 & $<0.001$ & 1.938 & 0.049 & 2.462 & 0.011 & 1.76 & 0.078 \\
\hline 根冠比 Root/shoot ratio & 0.976 & 0.461 & 0.099 & 0.753 & 6.005 & 0.015 & 0.479 & 0.49 & 1.099 & 0.365 & 0.855 & 0.567 & 1.093 & 0.369 \\
\hline
\end{tabular}

www.plant-ecology.com 

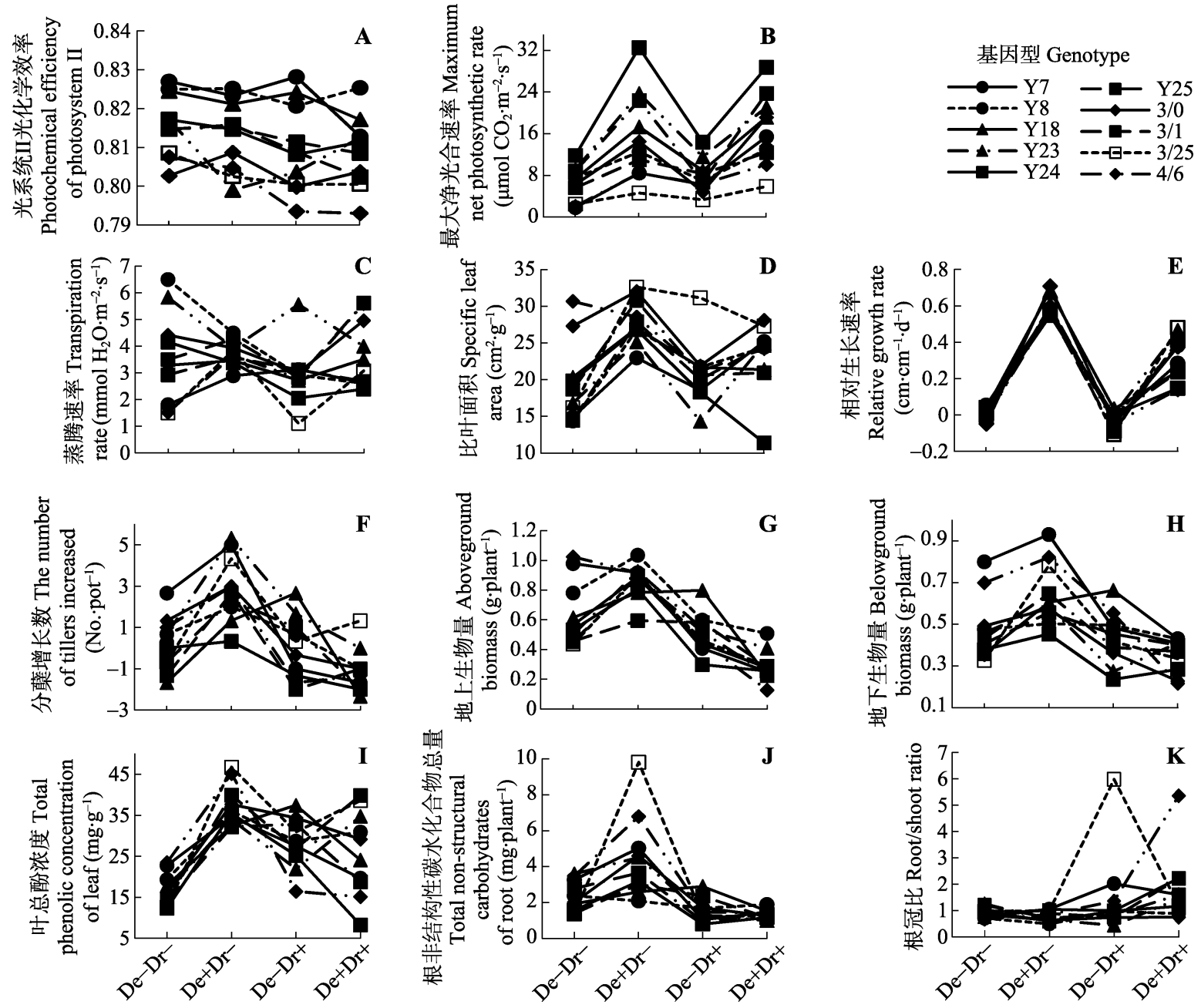

图1 四种处理条件下 10 个基因型羊草的反应规范。De-Dr-，对照; De+Dr-，非干旱刈割; De-Dr+, 非刈割干旱; De+Dr+，刏 割干旱。

Fig. 1 Reaction norms of traits of 10 Leymus chinensis genotypes under four treatments. De-Dr-, control; De+Dr-, non-arid defoliation; De-Dr+, non-defoliation but drought; De+Dr+, defoliation and drought.

\section{2 单因素方差分析及广义遗传力}

分别对4种处理条件下的性状平均值进行基因 型间的差异分析, 结果显示, 8 个性状在基因型间存 在显著差异 $(p<0.05)$, 而分藥增长数、地下部生物 量和TNC总量在 4 种环境条件下均未检测到基因型 间的差异 $(p>0.05)$ (表2)。在对照(De-Dr-)条件下, 6 个性状表现出基因型间的显著差异 $(p<0.05)$, 其中 $P_{\operatorname{nmax}}$ 和 $T_{\mathrm{r}}$ 的 $H^{2}$ 高于 0.5 ; 在刈割( $(\mathrm{De}+\mathrm{Dr}-)$ 条件下, 只 有 3 个性状表现出基因型间的显著差异, 其中 $P_{\mathrm{nmax}}$ 和 $R G R$ 的遗传力 $\left(H^{2}\right)$ 高于 0.5 ; 而干旱 $(\mathrm{De}-\mathrm{Dr}+$ )条件 下，5个性状表型表现出基因型间的显著差异 $(p<$ $0.05)$, 其中 $F_{\mathrm{v}} / F_{\mathrm{m}} 、 P_{\mathrm{nmax}}$ 和 $R G R$ 的 $H^{2}$ 高于 0.5 ; 刈割干 旱(De+Dr+)处理条件下，6个性状表现出基因型间 的显著差异 $(p<0.05)$, 其中 $F_{\mathrm{v}} / F_{\mathrm{m}} 、 P_{\mathrm{n} \text { max }}$ 和RGR的 $H^{2}$ 高于 0.5 。叶总酚浓度和根冠比仅在刈割干旱
$(\mathrm{De}+\mathrm{Dr}+)$ 条件下表现出基因型间的显著差异, $H^{2}$ 分 别为 0.145 和 0.202 。

\section{3 判别分析}

4 种处理的 $\mathrm{CDA}$ 表明, 两个函数就能解释总变 异的 59\%以上，但每种处理下，不同基因型羊草的 总体表型存在显著差异, 并且对前两个函数贡献较 大的指标也不完全相同; 另外，我们还可以发现， 无论是在哪种处理条件下, 同一个基因型的几个重 复的点在图中相距很近, 但并不能单独聚在一起, 表明环境和遗传因素均可以对羊草的表型变异产生 影响(表3; 图2)。

\section{3 讨论}

自然种群由表型多样的个体组成, 环境和遗传 均可以产生表型变异并驱动种群进化(Bolnick et al., 
表2 四种处理条件下羊草表型变异的单因素方差分析与广义遗传力 $\left(H^{2}\right)$ 分析

Table 2 ANOVA analysis and the broad-sense heritability $\left(H^{2}\right)$ estimates for 10 traits of phenotypic variations under four treatments in Leymus chinensis

\begin{tabular}{|c|c|c|c|c|c|c|c|c|}
\hline \multirow[t]{2}{*}{ 性状 Trait } & \multicolumn{2}{|c|}{ 对照 Control } & \multicolumn{2}{|c|}{ 刈割 Defoliation } & \multicolumn{2}{|c|}{ 干旱 Drought } & \multicolumn{2}{|c|}{$\begin{array}{c}\text { 刈割干早 } \\
\text { Defoliation and drought }\end{array}$} \\
\hline & $H^{2}$ & $p$ & $H^{2}$ & $p$ & $H^{2}$ & $p$ & $H^{2}$ & $p$ \\
\hline $\begin{array}{l}\text { 光系统II光化学效率 Photochemical efficiency of } \\
\text { photosystem II }\end{array}$ & 0.000 & $<0.001$ & 0.000 & $<0.001$ & 1.000 & $<0.001$ & 1.000 & $<0.001$ \\
\hline 最大净光合速率 Maximum net photosynthetic rate & 0.847 & $<0.001$ & 0.679 & $<0.001$ & 0.585 & $<0.001$ & 0.660 & $<0.001$ \\
\hline 蒸腾速率 Transpiration rate & 0.538 & $<0.001$ & - & 0.948 & 0.380 & $<0.001$ & 0.168 & 0.029 \\
\hline 比叶面积 Specific leaf area & 0.221 & 0.022 & - & 0.127 & 0.171 & 0.022 & - & 0.061 \\
\hline 相对生长速率 Relative growth rate & 0.400 & 0.002 & 0.727 & $<0.001$ & 0.625 & 0.001 & 0.515 & 0.006 \\
\hline 分暞增长数 The number of tillers increased & - & 0.314 & - & 0.286 & - & 0.410 & - & 0.056 \\
\hline 地上生物量 Aboveground biomass & 0.269 & 0.007 & - & 0.787 & - & 0.752 & - & 0.075 \\
\hline 地下生物量 Belowground biomass & - & 0.151 & - & 0.145 & - & 0.523 & - & 0.553 \\
\hline 叶总酚浓度 Total phenolic concentration of leaf & - & 0.170 & - & 0.201 & - & 0.616 & 0.145 & 0.004 \\
\hline $\begin{array}{l}\text { 根非结构性碳水化合物总量 Total non-structural } \\
\text { carbohydrates content of root }\end{array}$ & - & 0.201 & - & 0.062 & - & 0.550 & - & 0.951 \\
\hline 根冠比 Root/shoot ratio & - & 0.936 & - & 0.061 & - & 0.068 & 0.202 & 0.017 \\
\hline
\end{tabular}

-, 基因型间差异不显著未进行广义遗传力 $\left(H^{2}\right)$ 计算。

-, the broad-sense heritability $\left(H^{2}\right)$ was not calculated because the $p$-value is bigger than 0.05 .

表3 四种处理条件下羊草性状的典则判别函数系数

Table 3 Function coefficients of canonical discriminant traits of Leymus chinensis for four treatments

\begin{tabular}{|c|c|c|c|c|c|c|c|c|}
\hline \multirow[t]{2}{*}{ 性状 Traits } & \multicolumn{2}{|c|}{ 对照 Control } & \multicolumn{2}{|c|}{ 刏割 Defoliation } & \multicolumn{2}{|c|}{ 干旱 Drought } & \multicolumn{2}{|c|}{$\begin{array}{c}\text { 刈割干旱 } \\
\text { Defoliation and drought }\end{array}$} \\
\hline & $\begin{array}{c}\text { 函数1 } \\
\text { Function } 1\end{array}$ & $\begin{array}{c}\text { 函数2 } \\
\text { Function } 2\end{array}$ & $\begin{array}{c}\text { 函数1 } \\
\text { Function } 1\end{array}$ & $\begin{array}{c}\text { 函数 } 2 \\
\text { Function } 2\end{array}$ & $\begin{array}{l}\text { 函数1 } \\
\text { Function } 1\end{array}$ & $\begin{array}{c}\text { 函数 } 2 \\
\text { Function } 2\end{array}$ & $\begin{array}{l}\text { 函数1 } \\
\text { Function } 1\end{array}$ & $\begin{array}{c}\text { 函数 } 2 \\
\text { Function } 2\end{array}$ \\
\hline $\begin{array}{l}\text { 光系统II光化学效率 Photochemical efficiency of } \\
\text { photosystem II }\end{array}$ & 0.891 & 0.966 & -0.510 & -0.191 & 1.585 & 0.717 & -0.193 & 0.312 \\
\hline 最大净光合速率 Maximum net photosynthetic rate & 1.285 & -0.462 & -0.905 & 0.865 & 1.231 & -1.609 & 0.783 & -0.672 \\
\hline 蒸腾速率 Transpiration rate & -0.561 & 0.125 & 0.491 & -0.213 & 0.576 & -0.827 & 1.319 & 0.098 \\
\hline 比叶面积 Specific leaf area & -0.692 & -0.533 & 0.542 & 0.219 & 1.341 & -0.835 & -0.042 & 1.452 \\
\hline 相对生长速率 Relative growth rate & -0.588 & 0.815 & 0.962 & 0.953 & 1.235 & 0.236 & 1.101 & 0.267 \\
\hline 分葟增长数 The number of tillers increased & -0.416 & 0.347 & -0.424 & -1.030 & -1.365 & 0.603 & -0.079 & 0.521 \\
\hline 地上生物量 Aboveground biomass & 1.114 & 0.571 & 1.919 & 2.834 & -0.579 & 3.541 & 1.323 & 2.44 \\
\hline 地下生物量 Belowground biomass & -1.81 & 0.104 & -1.614 & -2.049 & 1.594 & -3.385 & 0.055 & -2.852 \\
\hline 叶总酚浓度 Total phenolic concentration of leaf & 0.126 & 0.905 & -0.714 & -0.021 & -0.115 & 0.486 & -1.226 & -1.07 \\
\hline $\begin{array}{l}\text { 根非结构性碳水化合物总量 } \\
\text { Total non-structural carbohydrates content of root }\end{array}$ & 0.598 & -1.076 & -0.010 & -0.446 & -1.058 & 0.564 & -0.129 & -0.498 \\
\hline 根冠比 Root/shoot ratio & 1.174 & 0.781 & 1.201 & 1.319 & -0.141 & 1.039 & 0.838 & 2.452 \\
\hline 解释的方差 Explained variance (\%) & 49.8 & 31.9 & 44.5 & 30.2 & 59.2 & 20.4 & 37.3 & 22.0 \\
\hline
\end{tabular}

2011)。在本研究中, 所观测指标在很大程度上均受 到环境条件的显著影响，这不仅表现在不同环境处 理间，也表现在同一环境处理条件下相同基因型间 (表1；图1，图2)，表明表型可塑性在羊草适应环境 中具有重要的作用。羊草表现出的较大的可塑性是 其能够耐受水分胁迫和放牧等外界环境、在不同生 境中广泛分布、大面积存在于欧亚草原的原因。可 塑性能够调节自然选择对植物的影响, 为植物面对 快速的气候变化提供一个缓冲，同时又能使其快速
适应新环境(Nicotra et al., 2010)。则割处理提高根部 $\mathrm{TNC}$ 含量(图1J), 即植株将光合产物更多地转移到 根部，为植株地上部分迅速再生提供物质基础，表 现出耐受性的再生; 在干旱及干旱刈割处理下，植 株根冠比增加(图1K), 以增加根部对水源的获取和 减少地上部对水分的蒸腾。然而，与水分充足时的 放牧相比，在干旱时期放牧，不同基因型羊草对环 境的防御性和耐受性都有不同程度的降低, 即干旱 降低了羊草对放牧的耐受性(Gao et al., 2008)和防 

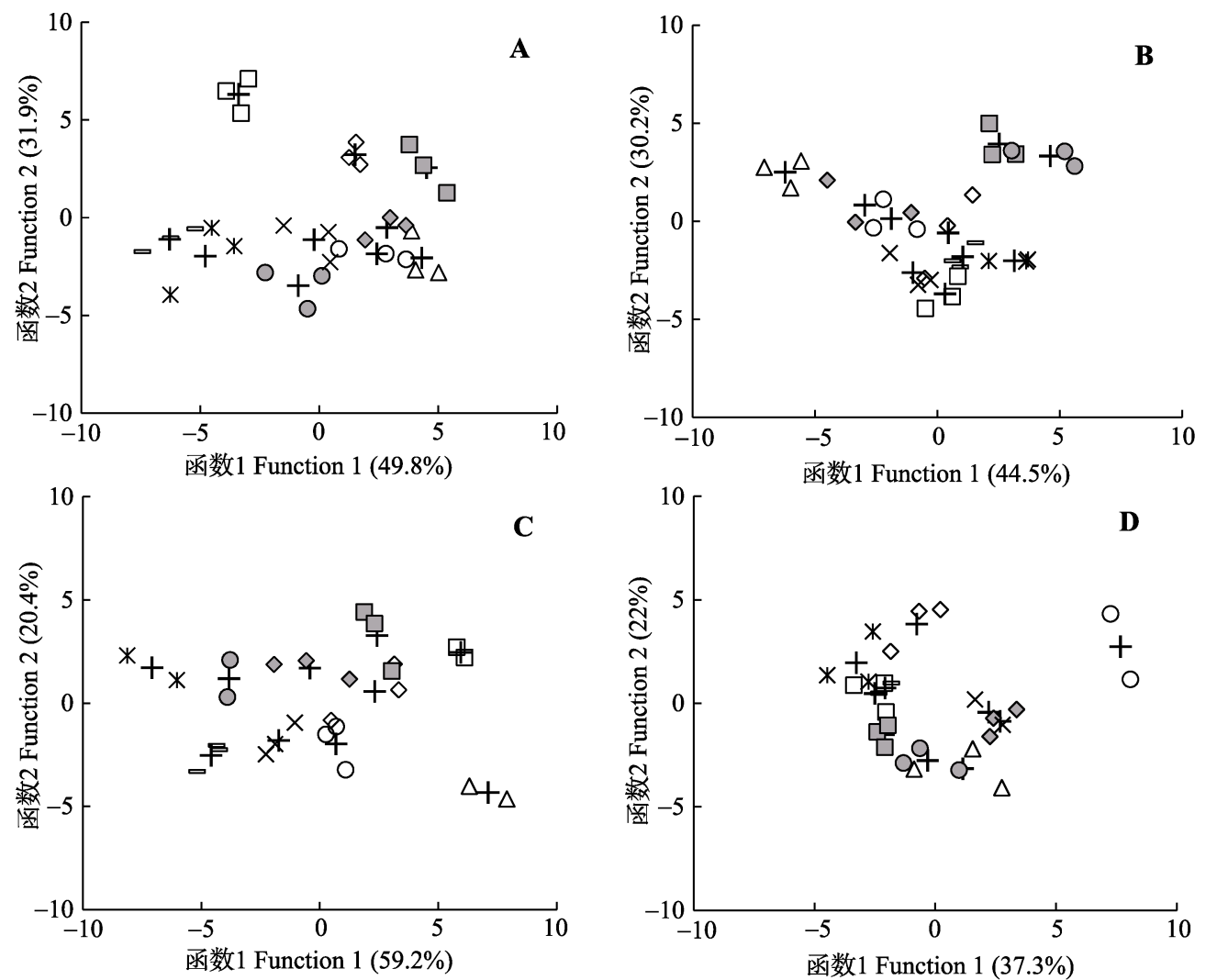

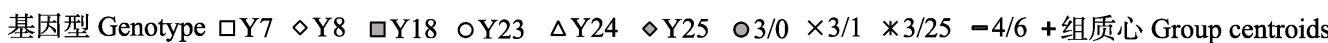

图2 四种处理下10个基因型羊草的典则判别函数散点图。A, 对照。 $\mathbf{B}$, 刈割。C, 干旱。

Fig. 2 Scatterplot of canonical discriminant of 10 genotypes in Leymus chinensis for four treatments. A, Control. B, Non-arid defoliation. C, Non-defoliation but drought. D, Defoliation and drought.

御性, 对种群的维持和功能的发挥产生不利影响。 类似报道, 如干旱胁迫导致苹果(Malus domestica) 树生长和产生的次级防御化合物浓度的下降 (Gutbrodt et al., 2012)。

除了受环境影响外, 多数生长指标受羊草基因 型的影响显著, 如 $P_{\mathrm{nmax}} 、 S L A 、 R G R$ 。羊草相对生长 速率和光合能力的种群内部差异(表1)说明不同基 因型羊草的资源获取能力不同(Yang et al., 2009), 从而导致种群内基因型间生态位的差异, 使得不同 基因型羊草能够以类似于物种间生态位互补的方式 共存, 维持自然种群中的多样性(Chang \& Smith, 2014)。这些结果合理地解释了申俊芳等(2015)研究 发现的多基因型组合羊草种群在提高种群生产力以 及抗干扰方面的正效应, 并深入分析得出互补效应 在其中起主导作用的结果。其他人的研究结果也支 持种内遗传多样性能够以与物种多样性相似的方式 提高群落生产力并维护其稳定性(Hughes et al., 2009; Kotowska et al., 2010)。
此外，基因型 $\times$ 刈割，基因型 $\times$ 干旱，以及基因 型 $\times$ 刈割 $\times$ 干旱对若干性状产生显著影响，表明环境 条件会对植物的适应机制及策略产生影响，这会进 一步影响基因型多样性功能的发挥, 且这种影响具 有遗传学基础。早有研究报道, 基因型间的分化更 倾向于在“胁迫”条件下而不是在“最佳”条件下表达 (Pigliucci et al., 1995)——随着胁迫水平的增加, 性 状的遗传力逐渐增加(Aslam et al., 2006); 但也有人 发现在干旱条件下性状遗传力会降低(Eid, 2009)。 另外, 性状的遗传力的大小也随环境条件变化且没 有一致的规律，但可以发现，仅在严重胁迫环境条 件(刚割干旱处理)下叶总酚浓度和根TNC总量表现 出基因型间的显著差异(表1，表2)，说明基因型的 生态适应策略发生了显著分化, 如基因型3/25用于 防御的能量增加，表现为叶总酚浓度显著升高，而 Y24中总酚浓度却非常低, 两者相差5倍左右。不同 基因型对环境变化响应的差异是普遍的, 如: 不同 基因型的Boechera stricta耐旱性差异显著(Haugen 
et al., 2008); 生长在不同地点的小麦(Triticum aestivum)的 26 个基因型的酚酸含量有很大差异 (Fernandez-Orozco et al., 2010); 不同基因型番茄 (Lycopersicon esculentum)在不同程度盐胁迫条件下, 根的生长, $\mathrm{Na} 、 \mathrm{~K}$ 含量以及相关蛋白组的表达不同 (Manaa et al., 2011)。Mallitt等(2010)研究发现, 在不 同水分和光照强度变化条件下, 不同基因型的 Lepidium bonariense 表现不同, 受基因型 $\times$ 水分 $\times$ 光 照交互作用影响显著。显著的基因型 $\times$ 环境交互作 用表明: 环境变化越复杂, 基因型越不可能以统一 的方式回应, 使得基因型多样性的存在能够维持种 群在变异的环境中生存(Ackerly et al., 2000), 对提 高群落稳定性或可恢复性十分重要。

羊草的表型差异不仅有广泛的可塑性基础, 而 且不同基因型羊草多数性状差异及性状可塑性变异 模式受遗传控制, 是其分布范围广、耐受性强的重 要原因, 也为其应对外界环境条件变化提供了保 障。结合建群种基因型多样性可以发挥类似物种水 平多样性的功能已有报道(Kotowska et al., 2010; Cook-Patton et al., 2011), 对羊草草原的保护不仅要 保护物种多样性, 也要保护羊草基因型多样性, 这 对草原生态系统的维持及退化草原的恢复和重建具 有重要的指导意义。

\section{基金项目 国家自然科学基金(31570427)。}

\section{参考文献}

Ackerly DD, Dudley SA, Sultan SE, Schmitt J, Coleman JS, Linder CR, Sandquist DR, Geber MA, Evans AS, Dawson TE (2000). The evolution of plant ecophysiological traits: Recent advances and future directions new research addresses natural selection, genetic constraints, and the adaptive evolution of plant ecophysiological traits. Bioscience, 50, 979-995.

Aslam M, KhanIA, Saleem M, Ali Z (2006). Assessment of water stress tolerance in different maize accessions at germination and early growth stage. Pakistan Journal of Botany, 38, 1571-1579.

Bolnick DI, Amarasekare P, Araújo MS, Bürger R, Levine JM, Novak M, Rudolf VH, Schreiber SJ, Urban MC, Vasseur DA (2011). Why intraspecific trait variation matters in community ecology. Trends in Ecology \& Evolution, 26, 183-192.

Chang CC, Smith MD (2014). Direct and indirect relationships between genetic diversity of a dominant grass, community diversity and above-ground productivity in tallgrass prai- rie. Journal of Vegetation Science, 25, 470-480.

Cook-Patton SC, McArt SH, Parachnowitsch AL, Thaler JS, Agrawal AA (2011). A direct comparison of the consequences of plant genotypic and species diversity on communities and ecosystem function. Ecology, 92, 915- 923.

Crutsinger GM, Sanders NJ, Classen AT (2009). Comparing intra-and inter-specific effects on litter decomposition in an old-field ecosystem. Basic and Applied Ecology, 10, 535-543.

Da Silveira AJ, Feitosa Teles F, Stull JW (1978). A rapid technique for total nonstructural carbohydrate determination of plant tissue. Journal of Agricultural and Food Chemistry, 26, 770-772.

Draghi JA, Whitlock MC (2012). Phenotypic plasticity facilitates mutational variance, genetic variance, and evolvability along the major axis of environmental variation. Evolution, 66, 2891-2902.

Eid MH (2009). Estimation of heritability and genetic advance of yield traits in wheat (Triticum aestivum L.) under drought condition. International Journal of Genetics and Molecular Biology, 1, 115-120.

Fernandez-Orozco R, Li L, Harflett C, Shewry PR, Ward JL (2010). Effects of environment and genotype on phenolic acids in wheat in the health grain diversity screen. Journal of Agricultural and Food Chemistry, 58, 9341-9352.

Gao Y, Wang D, Ba L, Bai Y, Liu B (2008). Interactions between herbivory and resource availability on grazing tolerance of Leymus chinensis. Environmental and Experimental Botany, 63, 113-122.

Gu WC (2004). Statistical Genetic. Science Press, Beijing. (in Chinese) [顾万春 (2004). 统计遗传学. 科学出版社, 北 京.]

Gutbrodt B, Dorn S, Mody K (2012). Drought stress affects constitutive but not induced herbivore resistance in apple plants. Arthropod-Plant Interactions, 6, 171-179.

Haugen R, Steffes L, Wolf J, Brown P, Matzner S, Siemens DH (2008). Evolution of drought tolerance and defense: Dependence of tradeoffs on mechanism, environment and defense switching. Oikos, 117, 231-244.

Hedrick PW (2005). A standardized genetic differentiation measure. Evolution, 59, 1633-1638.

Hu BZ, Liu D, Hu GF, Jiang SJ, Zhang AY (2001). Morphological variation and genetic diversity in Aneurolepidium chinensis. Acta Phytoecologica Sinica, 25, 83-89. (in Chinese with English abstract) [胡宝忠, 刘娣, 胡国富, 姜述君, 张阿英 (2001). 羊草遗传多样性的研究. 植物 生态学报, 25, 83-89.]

Hughes AR, Stachowicz JJ, Williams SL (2009). Morphological and physiological variation among seagrass (Zostera marina) genotypes. Oecologia, 159, 725-733. 
Johnson MT, Agrawal AA (2005). Plant genotype and environment interact to shape a diverse arthropod community on evening primrose (Oenothera biennis). Ecology, 86, 874-885.

Joshi J, Stoll P, Rusterholz HP, Schmid B, Dolt C, Baur B (2006). Small-scale experimental habitat fragmentation reduces colonization rates in species-rich grasslands. Oecologia, 148, 144-152.

Kanaga MK, Ryel RJ, Mock KE, Pfrender ME (2008). Quantitative-genetic variation in morphological and physiological traits within a quaking aspen (Populus tremuloides) population. Canadian Journal of Forest Research, 38, 16901694.

Kotowska AM, Cahill JJ, Keddie BA (2010). Plant genetic diversity yields increased plant productivity and herbivore performance. Journal of Ecology, 98, 237-245.

Lande R (2009). Adaptation to an extraordinary environment by evolution of phenotypic plasticity and genetic assimilation. Journal of Evolutionary Biology, 22, 1435-1446.

Le Corre V, Kremer A (2012). The genetic differentiation at quantitative trait loci under local adaptation. Molecular Ecology, 21, 1548-1566.

Li H, Yang YF, Lu XS (2004). Quantitative analysis of reproductive tiller characteristics of Leymus chinensis populations on the Songnen Plain of China. Acta Prataculturae Sinica, 13(4), 50-56. (in Chinese with English abstract) [李红, 杨允菲, 卢欣石 (2004). 松嫩平原羊草种群生 殖分藥株的数量特征及其定量分析. 草业学报, 13(4), 50-56.]

Li HY, Li JD, Xu ZG, Zhou JY, Zhang JF (2011). Vegetative reproduction characteristics of Leymus chinensis populations in Tumuji National Nature Reserve, Inner Mongolia. Acta Prataculturae Sinica, 20(5), 19-25. (in Chinese with English abstract) [李海燕, 李建东, 徐振国, 周景英, 张 建峰 (2011). 内蒙古图牧吉自然保护区羊草种群营养 繁殖特性的比较. 草业学报 20(5), 19-25.]

Liefting M, Hoffmann AA, Ellers J (2009). Plasticity versus environmental canalization: Population differences in thermal responses along a latitudinal gradient in Drosophila serrata. Evolution, 63, 1954-1963.

Liu HF, Gao YB, Ruan WB, Chen L, Li CL, Zhao NX, Wang D (2004a). Genetic differentiation within and between Leymus chinensis populations from different zones of mid-eastern Inner Mongolia steppe. Acta Ecologica Sinica, 24, 2157-2164. (in Chinese with English abstract) [刘惠 芬, 高玉葆, 阮维斌, 陈磊, 李长林, 赵念席, 王丹 (2004a). 内蒙古中东部不同草原地带羊草种群遗传分 化. 生态学报, 24, 2157-2164.]

Liu HF, Gao YB, Wang D, Ren AZ, Ruan WB, Chen L, Zhao NX (2004b). Genetic differentiation in eight populations of Leymus chinensis in Inner Mongolia steppe. Acta Ecologica Sinica, 24, 423-431. (in Chinese with English abstract) [刘惠芬, 高玉葆, 王丹, 任安芝, 阮维斌, 陈 磊, 赵念席 (2004b). 内蒙古典型草原羊草种群遗传分 化的RAPD分析. 生态学报, 24, 423-431.]

Malinowski DP, Alloush GA, Belesky DP (1998). Evidence for chemical changes on the root surface of tall fescue in response to infection with the fungal endophyte Neotyphodium coenophialum. Plant and Soil, 205, 1-12.

Mallitt KL, Bonser SP, Hunt J (2010). The plasticity of phenotypic integration in response to light and water availability in the pepper grass, Lepidium bonariense. Evolutionary Ecology, 24, 1321-1337.

Manaa A, Ahmed HB, Valot B, Bouchet JP, Aschi-Smiti S, Causse M, Faurobert M (2011). Salt and genotype impact on plant physiology and root proteome variations in tomato. Journal of Experimental Botany, 17, 1-17.

Miner BG, Sultan SE, Morgan SG, Padilla DK, Relyea RA (2005). Ecological consequences of phenotypic plasticity. Trends in Ecology \& Evolution, 20, 685-692.

Nicotra AB, Atkin OK, Bonser SP, Davidson AM, Finnegan E, Mathesius U, Poot P, Purugganan MD, Richards C, Valladares F (2010). Plant phenotypic plasticity in a changing climate. Trends in Plant Science, 15, 684-692.

Pigliucci M, Schlichting C, Whitton J (1995). Reaction norms of Arabidopsis. II. Response to stress and unordered environmental variation. Functional Ecology, 9, 537-547.

Richards JH (1993). Physiology of Plants Recovering from Defoliation. SIR Publishing, Wellington, New Zealand. 85-94.

Seliskar DM, Gallagher JL, Burdick DM, Mutz LA (2002). The regulation of ecosystem functions by ecotypic variation in the dominant plant: A Spartina alterniflora salt-marsh case study. Journal of Ecology, 90, 1-11.

Shen JF, Ren HQ, Xin XJ, Xu B, Gao YB, Zhao NX (2015). Leymus chinensis genotypic diversity increases the response of population to disturbance. Acta Ecologica Sinica, 35, 7682-7689. (in Chinese with English abstract) [申俊芳, 任慧琴, 辛晓静, 徐冰, 高玉葆, 赵念席 (2015). 羊草基因型多样性能增强种群对干扰的响应. 生态学报, 35, 7682-7689.]

Stevens MT, Waller DM, Lindroth RL (2007). Resistance and tolerance in Populus tremuloides: Genetic variation, costs, and environmental dependency. Evolutionary Ecology, 21, 829-847.

Strasburg JL, Sherman NA, Wright KM, Moyle LC, Willis JH, Rieseberg LH (2012). What can patterns of differentiation across plant genomes tell us about adaptation and speciation? Philosophical Transactions of the Royal Society of London B: Biological Sciences, 367, 364-373. 
Sultan SE (1995). Phenotypic plasticity and plant adaptation. Acta Botanica Neerlandica, 44, 363-383.

Sultan SE (2000). Phenotypic plasticity for plant development, function and life history. Trends in Plant Science, 5, $537-542$.

Toker C (2004). Estimates of broad-sense heritability for seed yield and yield criteria in faba bean (Vicia faba L.). Hereditas, 140, 222-225.

Wang D (2004). The Variance and Differentiation of Leymus chinensis in Mid-Eastern Inner Mongolia Grassland. Master degree dissertation, Nankai University, Tianjin. 35-45. (in Chinese with English abstract) [王丹 (2004).
内蒙古中东部草原羊草种内变异与分化研究. 硕士学 位论文，南开大学，天津. 35-45.]

Wang R, Gao Q (2001). Photosynthesis, transpiration, and water use efficiency in two divergent Leymus chinensis populations from Northeast China. Photosynthetica, 39, 123-126.

Yang Y, Fang J, Ji C, Han W (2009). Above- and belowground biomass allocation in Tibetan grasslands. Journal of Vegetation Science, 20, 177-184.

责任编委: 陈小勇 责任编辑: 李 敏

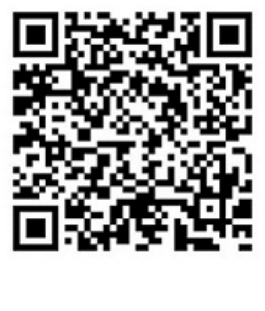

